\title{
Measurement and Simulation of Microwave Noise Transient of InP/InGaAs DHBT with Polyimide Passivattion
}

\author{
Yong Zhong Xiong ${ }^{*}$, Geok-Ing Ng, Hong Wang, Jeffrey S. Fu, and Radhakrishnan K \\ Microelectronics Centre, School of Electrical and Electronics Engineering, Nanyang Technological \\ University, Singapore 639798 *Corresponding author, Tel: (65) 7904234, Fax: (65) 7933318, e-mail: \\ eyzxiong@ntu.edu.sg
}

\begin{abstract}
Measurement and simulation of microwave noise transient of InP/InGaAs DHBT with polyimide passivation is reported in this paper for the first time and is believed to contribute to the overall broadband shot noise. This work provides a better insight into the noise transient mechanism of InP HBTs due to polyimide passivation and can be used to improve the device and circuit reliability.
\end{abstract}

\section{INTRODUCTION}

In recent years, many investigations have been conducted to study the effects of surface passivation on the performance of InP-based HBTs [1][2][3]. The device passivation and protection for InP HBTs rely on the dielectric films such as silicon nitride, silicon oxide or polyimide. For InP-based HBTs, polyimide was found to induce the least degradation as compared to $\mathrm{SiN}_{\mathrm{X}}$ and $\mathrm{SiO}_{2}$ [4], and thus has been widely used for passivation and planarization of InP HBTs $[5,6]$. However, we have recently observed a current transient and current gain instabilities in $\mathrm{InP} / \mathrm{InGaAs}$ HBTs induced by polyimide passivation layer [7]. The causes of the current transient have been carefully investigated. It was found out that, in the HBTs covered by polyimide, static charges may accumulate in the polyimide film or at the interface. The trapping and detrapping of static charge in the polyimide at the interface causes the change of surface potential at the base-emitter junction perimeter causing base and collector current transients. However, in [7], the studies were limited to the effect of DC characteristics such as base current and DC gain. No investigation was made for the effect of the polyimide passivation on the device microwave performance. For example, the change of surface potential induced by electron trapping and de-trapping may induce the change of the wide-band shot noise.

This paper describes the measurement of microwave noise transient of InP-based HBTs. Moreover, in order to gain a fundamental understanding of the effect of current transients on the noise characteristics, a simulation method for noise figure transient is also presented. The change of dc base and collector currents caused by the trapping \& de-trapping in the polyimide film were measured under higher collector current. A function of second order exponential decay with two-timeconstants was used to fit the transient currents. And the noise figures transient measurements were demonstrated using the commercial noise measurement system NP5. The simulated transient noise results, which were obtained based on the measured dc transient currents, are also presented. As noise transients of devices are harmful for the stability of circuits in many communication systems, the understanding of the mechanism for the noise transient of the InP HBTs is very useful to improve the reliability of the devices and circuits.

\section{DC CURRENT TRANSIENT MEASURMENT}

The InP DHBT device with $5 \times 10 \mu \mathrm{m}^{2}$ emitters and self-aligned base electrodes were investigated under room temperature. Device dc characteristics and current transient were measured using a HP 4156B semiconductor parameter analyzer and cascade microwave probe.

\section{A. Base Current Transient}

The phenomenon and its physical mechanism of the base current transient were presented in [7]. For a device with constant base and collector voltage biases, the intrinsic base and collector currents may instantaneously reach its steady value when the device is turned on. However, the total base current $\left(I_{B}=I_{B 0}+I_{B e}(t)\right)$ decays slowly due to the decrease of excess base current induced by the change of the electric field in the polyimide caused by the electron detrapping. This will result in the decrease in the corresponding noise figure due to a decrease in the wide-band shot noise with the decrease of the transient dc-current. To study the dc-current transient effect on the noise figure transient, the dc-current transients of the HBT were measured. Fig. 1 shows the base current transient with emitter size of $5 \times 10 \mu \mathrm{m}^{2}$ at $V_{B E}=0.665 \mathrm{~V}$, and $0.685 \mathrm{~V}$ and the base-collector voltage $\left(V_{B C}\right)$ was fixed at $-1.0 \mathrm{~V}$ and each measurement was set to last 27.5 seconds and step time of $0.67 \mathrm{~s}$. In order to make sure the empty traps can be fully recharged, an interruption time as long as $1200 \mathrm{~s}$ was set between measurements. It is obvious from Fig. 1 that the base current increases with increasing base voltage $\left(\mathrm{V}_{\mathrm{BE}}\right)$, while the time 
taken for the base current to decay to steady state becomes shorter. This indicates that the electron detrapping from the polyimide traps may have an electrical field dependence, which provides evidence that the electron detrapping could be through tunneling process [7]. In fact, it has been found that the electrons are distributed in the polyimide film within the range of $1 \mathrm{~nm}$ from the interface. When base bias voltage increases, the electric field near the base-emitter junction perimeter at the polyimide-emitter interface in the polyimide films increases. Thus the time for the trapped electrons to escape from the polyimide decreases thus induce a shorter detrapping transient time constant. Assuming the trapped electrons distributed in the polyimide film with different distance from interface have different detrapping time constants, thus the base current transient should obey multi-order exponential decay, e.g. multi-time-constant. In order to simplify the calculation, the function of second order exponential decay with two-time-constants was selected to fit the current transient [8]. The expression is given by

$$
I_{B}(t)=I_{B 0}+I_{B 1} e^{-\frac{t}{\tau_{1}}}+I_{B 2} e^{-\frac{t}{\tau_{2}}}
$$

where $I_{B}(\mathrm{t})$ is the total base current; $I_{B 0}$ is the steady state base current; $I_{B 1}$, and $I_{B 2}$ are the first order and second order base detrapping transient currents, respectively; and $\tau_{1}$ and $\tau_{2}$ are the first order and second order base detrapping transient time constants, respectively. We can see that the experimental data can be fitted very well by using the two-time constants approach.

\section{B. Collector Current Transient}

In the InP-HBTs, the device junction surfaces are covered by polyimide, the charge exchange may happen at the polyimide/semiconductor interface. The net result of the charge exchange is the accumulation of the charge near the basecollector junction. These result in the surface potential of the sidewall of base-collector junction. It is noted that the inversion occurs on the surface of lower n-type doping concentration of collector $\left(5 \times 10^{15} \mathrm{~cm}^{-3}\right)$ under a surface potential. These indicate that there is a parasitic polyimide field induced leakage path along the surface of the base-collector [9]. This leakage patch can be treated as a parasitic ployimide field effect transistor (ppFET), and the effective channel length of this device is close to the depletion width of the base-collector junction. The root cause of the change in the surface potential on the sidewall of the base-collector is similar to that of base-emitter junction. The surface potential is related to the electron trap density in the polyimide film as well as the bias voltages. When the base-emitter voltage of the device increases, the surface field near the base-collector which causes the electrons detrapping from polyimide traps may also increase, thereby results in the difference in the time constants.

Fig. 2 shows the collector current versus time with emitter size of $5 \times 10 \mu \mathrm{m}^{2}$ at $V_{B E}=0.665 \mathrm{~V}$ and $0.685 \mathrm{~V}$, respectively, and the base-collector voltage $\left(V_{B C}\right)$ was fixed at $-1.0 \mathrm{~V}$. Again, we also selected the function of second order exponential decays to fit the curves of the base current transient. The expression is given by:

$$
I_{C}(t)=I_{C 0}+I_{C 1} e^{-\frac{t}{\tau_{1}}}+I_{C 2} e^{-\frac{t}{\tau_{2}}}
$$

where $I_{C}(\mathrm{t})$ is total base current; the $I_{C 0}$ is the steady state collector current; $I_{C l}$, and $I_{C 2}$ are the first order and second order collector spurious transient currents, respectively.

\section{MICROWAVE NOISE TRANSIENT MEASUREMENT}

The microwave noise parameters were measured on-wafer from 2-20 GHz using an ATN-NP5 Noise Parameter Measurement system, an HP8970C noise figure test set, an HP8970B noise figure meter, and an HP8510C network analyzer. The noise figure and associated gain of the device under test were measured at various impedance matching and DC bias conditions. Next, the minimum noise figure, noise resistance and the optimal source impedance $\left(N F_{M I N}, R_{N}, G_{O P T}\right.$ and $B_{O P T}$ ) were extracted from the measured noise figures at a particular DC bias.

As it commonly known, the change of DC-currents results in the change of noise figure. Thus the DC-current transient will result in the noise figure transient. Therefore, to accurately measure the steady state S-parameters and noise parameters, the interruption time between two measurements was set to as long as 1200 seconds for the device to stabilize under constant base and collector biasing voltages. The steady state noise parameters $\left(N F_{M I N}, R_{N}, G_{O P T}\right.$ and $\left.B_{O P T}\right)$ with emitter size of $5 \mathrm{x} 10 \mu \mathrm{m}^{2}$ at different constant voltage bias conditions were measured. The optimal source impedance $\left(0.622 \angle 31.3^{\circ}\right)$ at frequency of $10 \mathrm{GHz}$ and $V_{B E}=0.685 \mathrm{~V}$ and $V_{C E}=1.5 \mathrm{~V}$ indicates that the value is very close to a given solid-state impedance point of the solid-state tuner $\left(0.620 \angle 29.0^{\circ}\right)$. At these bias conditions, the steady state minimum noise figure is about $\sim 1.9$ $(2.78 \mathrm{~dB})$, the steady state associated gain is about $\sim 5.5 \mathrm{~dB}$ and the steady state noise resistance is about $\sim 76.5 \Omega$. Thus, the existing solid-state impedance $\left(0.620 \angle 29.0^{\circ}\right)$ was chosen as the source impedance under user pro-selected mode in ATN NP5 for the same biasing condition. After turning on the device and waited as long as 1200s for making sure the empty traps can be fully recharged, the s-parameters measurements were made followed by the noise figures measurement and then the device was turned off before starting the next set of measurement. It is noted that the ATN NP5 software needs a time of $0.67 \mathrm{~s}$ for each noise figure measurement. The measured results indicated the noise figure decreased from 2.1 (3.22 $\mathrm{dB})$ to $1.9(2.78 \mathrm{~dB})$ with the time from $2.01 \mathrm{~s}$ to $27.48 \mathrm{~s}$ and the step time is about $0.67 \mathrm{~s}$, but the associated gain was almost maintained at $\sim 5.5 \mathrm{~dB}$. The measured optimal source impedance at frequency of $10 \mathrm{GHz}$ and $V_{B E}=0.665 \mathrm{~V}$ and $V_{C E}=1.5 \mathrm{~V}$ 
is $0.630 \angle 42.3^{\circ}$. This is also very close to the solid-state impedance of the solid-state tuner $\left(0.620 \angle 29.0^{\circ}\right)$ and the noise figure decreased from $2.7(4.31 \mathrm{~dB})$ to $2.1(3.22 \mathrm{~dB})$. Fig. 3 shows the time dependence of transient noise figures at different $V_{B E}=0.665 \mathrm{~V}$, and $0.685 \mathrm{~V}$, respectively, and $V_{B C}=-1.0 \mathrm{~V}$ for emitter size of $5 \mathrm{x} 10 \mu \mathrm{m}^{2}$. It is obvious that the time constant of noise figure transient at lower base voltage $\left(V_{B E}\right)$ is longer than the one of higher $\left(V_{B E}\right)$.

\section{SIMULATION RESULTS}

Under constant voltage bias conditions, the intrinsic device reaches the steady state very fast. But the excess transient currents on the surfaces of the base-emitter and the sidewall of the base-collector induced by the surface potential reach to them highest values very fast, respectively, then decay slowly. Therefore, the device noise is consisting of the steady state current shot noises, the thermal noises and the excess base and collector transient current shot noises. Following the noise calculation procedure of [10], the minimum noise figure can be calculated. Fig. 3 shown that excellent agreement was obtained between the measured and simulated transient results. This indicates that it is more accurate and convenient to predict the transient of the noise figure based on the steady-state small-signal equivalent circuits and the transient DC-base and collector transient currents.

\section{SUMMARY}

The noise transient of InP HBT with polyimide passivation was investigated experimentally under constant voltage bias conditions at $10 \mathrm{GHz}$. The base and collector transient currents induced by the surface potential in the polyimide film were measured for the transient shot noise calculation. The simulated results are in good agreement with the measured results. The time dependence of microwave noise figures due to the excess transients is investigated. We have also demonstrated that the experimental results can be well predicted by simulations. As to the selection of the bias conditions for device applications, tradeoffs between the noise transient and the noise figure should be taken into account. This work provides a better insight into the noise transient mechanism of InP HBTs due to polyimide passivation and can be used to improve the device and circuit reliability.

\section{ACKNOWLEDGMENT}

The authors wish to thank H.Q. Zheng, C. L.Tan, for their helpful discussions. This work is supported by the grant under the research and development project of the National Science and Technology Board of Singapore.

\section{REFERENCES}

[1] E. Tokumitsu, A. G. Dentai, C. H. Joyner, "Reduction of the surface recombination current in InGaAs/InP pseudoheterojunction bipolar transistors using a thin InP passivation layer," IEEE Electron Device Lett., vol.10, pp585-587, 1989.

[2] D. Sachelarie, J. L. Pelouard, J. L.Benchimol, "Orientation effect on $\mathrm{Si}_{3} \mathrm{~N}_{4}$ passivated $\mathrm{InP} / \mathrm{InGaAs}$ heterojunction bipolar transistors," Eighth International Conference on Indium Phosphide and Related Materials, pp.149 -151, 1996.

[3] A. Ouacha, M. Willander, B. Hammarlund, and R. A. Logan,"Effect of surface passivation with SiN on the electrical properties of InP/InGaAs heterojunction bipolar transistors," J. Appl. Phys., vol.74, pp.5602-5605, 1993.

[4] D. Caffin, L. Bricard, J. L. Courant, L. S. How Kee Chun, B. Lescaut, A.M. Duchenois, M. Meghelli, J. L. Benchimol, and P. Launay, "Passivation of InP-based HBTs for high bit rate circuit applications," International Conference on Indium Phosphide and Related Materials, pp.637 -640, 1997.

[5] S.-I. Tanaka, K. Kashahara, H. Shimawaki, and K. Honjo, "Stress current behavior of InAlAs/InGaAs and AlGaAs/GaAs HBT's with polyimide passivation," IEEE Electron Device Lett., vol.13, pp.560-562, 1992.

[6] S. Hyunchol, C. Gaessler, and H. Leier, "Reduction of base-collector capacitance in InP/InGaAs HBT's using a novel double polyimide planarization process," IEEE Electron Device Lett., vol.19, pp.297-299, 1998.

[7] Hong Wang and G. I. Ng " Current Transient in Polyimide-Passivated InP/InGaAs Heterojunction Bipolar Transistors: Systematic Experiments and Physical Model " IEEE Electron Devices, Vol. 47, pp2261-2269, Dec. 2000

[8] Tahui Wang; Chang, T.E.; Chiang, L.P.; Huang, C. "A new technique to extract oxide trap time constants in MOSFET's" IEEE Electron Device Letters Volume: 178 pp398 -400, Aug. 1996.

[9] Hook, T.B. "Polyimide-related design considerations in a bipolar technology" IEEE Transactions on Electron Devices, Volume: 377 , pp1714 -1718, July 1990.

[10] Rouquette, P.; Gasquet, D.; Holden, T.; Moult, J. "HBT's RF noise parameter determination by means of an efficient method based on noise analysis of linear amplifier networks" IEEE Transactions on Microwave Theory and Techniques, Vol45, pp690 -694, May 1997. 


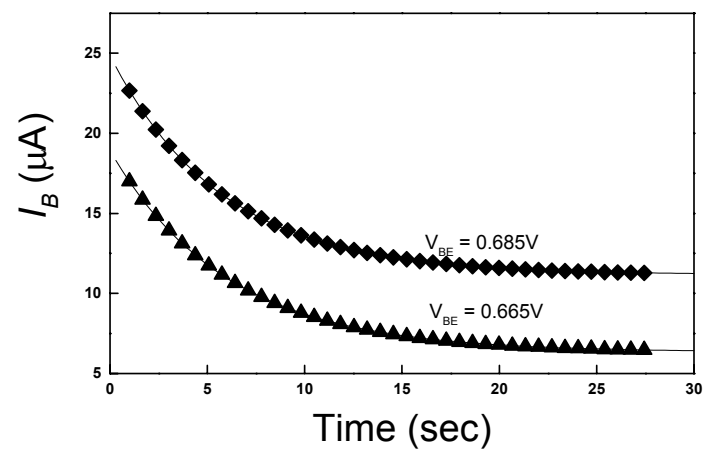

Fig. 1 The base current against time with emitter size $\left(5 \times 10 \mu \mathrm{m}^{2}\right)$ at $V_{B E}=0.665 \mathrm{~V}$ and $0.685 \mathrm{~V}$ and the base-collector voltage $\left(V_{B C}\right)$ was fixed at $-1.0 \mathrm{~V}$ (the symbol lines are the measured data and the solid lines are the fit data).

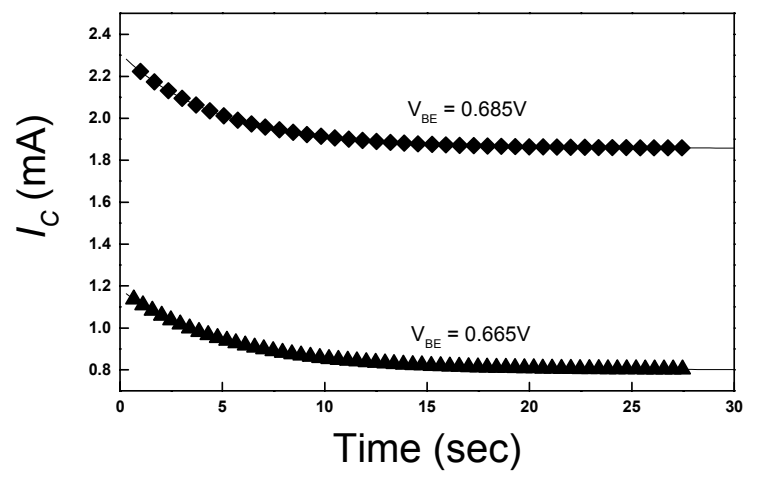

Fig. 2 The collector current against time with emitter size $\left(5 \times 10 \mu \mathrm{m}^{2}\right)$ at $V_{B E}=0.665 \mathrm{~V}$ and $0.685 \mathrm{~V}$ and the base-collector voltage $\left(V_{B C}\right)$ was fixed at $-1.0 \mathrm{~V}$ (the symbol lines are the measured data and the solid lines are the fit data).

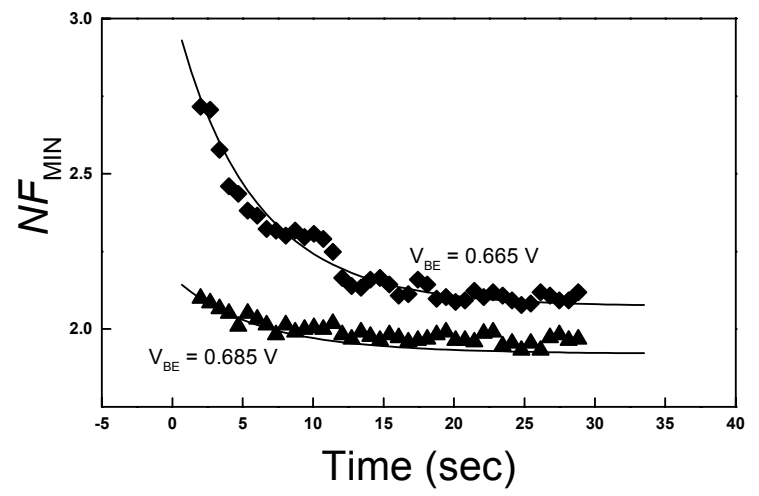

Fig.3 The time dependence of transient noise figures at the different $V_{B E}=0.665 \mathrm{~V}$, and $0.685 \mathrm{~V}$ and $V_{C E}=1.5 \mathrm{~V}$ for emitter size of $5 \times 10 \mu \mathrm{m}^{2}$ (the symbol lines are the measured data and the solid lines are the fit data). 